
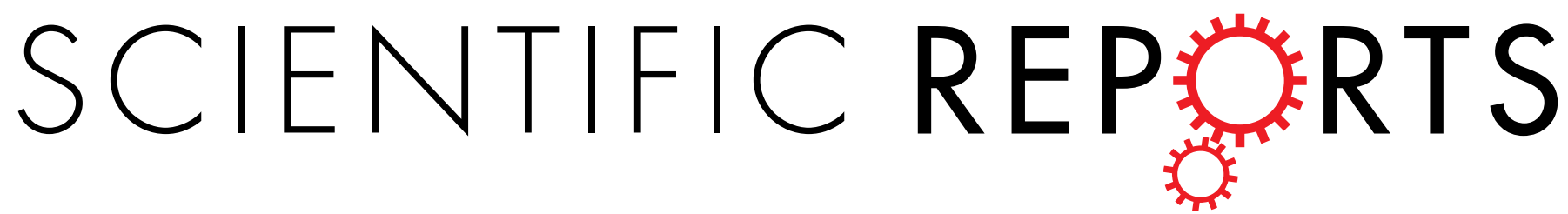

\title{
OPEN The role of GRIP1 and ephrin B3 in blood pressure control and vascular smooth muscle cell contractility
}

Received: 24 June 2016

Accepted: 16 November 2016

Published: 12 December 2016
Yujia Wang ${ }^{1}$, Zenghui Wu ${ }^{1}$, Hongyu Luo ${ }^{1}$, Junzheng Peng ${ }^{1}$, John Raelson ${ }^{1}$, Georg B. Ehret ${ }^{2}$, Patricia B. Munroe ${ }^{3}$, Ekatherina Stoyanova ${ }^{1}$, Zhao Oin $^{1}$, Guy Cloutier ${ }^{1}$, W. Edward Bradley ${ }^{1}$, Tao $\mathrm{Wu}^{4}$, Jian-Zhong Shen ${ }^{4}$, Shenjiang $\mathrm{Hu}^{4}$ \& Jiangping Wu ${ }^{1,5}$

Several erythropoietin-producing hepatocellular receptor B family (EPHB) and their ligands, ephrinBs (EFNBs), are involved in blood pressure regulation in animal models. We selected 528 single nucleotide polymorphisms (SNPs) within the genes of EPHB6, EFNB2, EFNB3 and GRIP1 in the EPH/EFN signalling system to query the International Blood Pressure Consortium dataset. A SNP within the glutamate receptor interacting protein 1 (GRIP1) gene presented a $p$-value of 0.000389 , approaching the critical $p$-value of 0.000302 , for association with diastolic blood pressure of 60,396 individuals. According to echocardiography, we found that Efnb3 gene knockout mice showed enhanced constriction in the carotid arteries. In vitro studies revealed that in mouse vascular smooth muscle cells, siRNA knockdown of GRIP1, which is in the EFNB3 reverse signalling pathway, resulted in increased contractility of these cells. These data suggest that molecules in the EPHB/EFNB signalling pathways, specifically EFNB3 and GRIP1, are involved blood pressure regulation.

Erythropoietin-producing hepatocellular receptor $(\mathrm{EPH})$ kinases are the largest family of receptor tyrosine kinases. They are divided into A and B subfamilies according to sequence homology ${ }^{1}$. Ephrins (EFNs), which are also cell surface molecules, are ligands of EPHs. EFNs are classified as A and B subfamilies. EFNAs attach to the cell surface through glycosylphosphatidylinositol anchoring, whereas EFNBs attach through transmembrane sequences $^{2-4}$. Interactions among EPHs and EFNs are promiscuous but, in general, EPHA members interface preferentially with EFNAs, and EPHBs with $\mathrm{EFNBs}^{2-4}$. Such redundancy suggests that these kinases are crucial in various biological contexts. EFNs can stimulate EPH receptors, and this is called forward signalling. Interestingly, EPHs are also capable of stimulating EFNs which then transmit signalling reversely into cells, a phenomenon known as reverse signalling.

EPHs and EFNs are expressed in many tissues and organs. They play important roles in the central nervous system $^{2,4}$, immune system ${ }^{5-14}$, digestive system ${ }^{15}$, bone metabolism ${ }^{16,17}$, angiogenesis ${ }^{18}$ and other processes ${ }^{19-21}$.

We recently reported that EPHB6, in concert with sex hormones, is crucial in VSMC contraction and blood pressure (BP) regulation ${ }^{22}$. Ephb6 gene knockout (KO) mice after castration manifest higher blood pressure that their wild type (WT) counterparts ${ }^{22}$. Vascular smooth muscle cells (VSMC) are a target tissue through which EPHB6 exerts its effect on BP control. Since EPHB6 and all its major ligands of the EFNB family, i.e., EFNB1, EFNB2 and EFNB3, are expressed in VSMCs ${ }^{22}$, there is a molecular framework for their function in these cells.

We showed that while solid-phase recombinant EPHB6 reduces VSMC contraction in response to phenylephrine (PE) stimulation, solid-phase anti-EPHB6 antibody $(\mathrm{Ab})$ does not $^{22}$, indicating that reverse signalling from EPHB6 to EFNBs but not forward signalling from EFNBs to EPHB6 is responsible for dampening VSMC contractility. Since all the cells in the vascular bed express EPHB6 and the 3 EFNB ligands, EPHB6 expressed in neighboring VSMCs can trigger reverse signaling of a certain EFNBs in a VSMC. For EPHB6, such reverse

${ }^{1}$ Research Centre, Centre hospitalier de I'Université de Montréal (CHUM), Montreal, Quebec H2X 0A9, Canada. ${ }^{2}$ Center for Complex Disease Genomics, McKusick-Nathans Institute of Genetic Medicine, Johns Hopkins University School of Medicine, Baltimore, Maryland 21205, USA. ${ }^{3}$ Clinical Pharmacology and The Genome Centre, William Harvey Research Institute, Barts and The London School of Medicine and Dentistry, Queen Mary University of London, London EC1M 6BO, UK. ${ }^{4}$ Institute of Cardiology, First Affiliated Hospital, Zhejiang University Medical College, Hangzhou, 310003, China. ${ }^{5}$ Nephrology Service, Centre hospitalier de I'Université de Montréal (CHUM), Montreal, Quebec H2X 0A9, Canada. Correspondence and requests for materials should be addressed to J.W. (email: jianping.wu@umontreal.ca) 


\begin{tabular}{|c|c|c|c|c|c|c|}
\hline \multicolumn{7}{|c|}{ Locations of 4 genes for which 528 SNPs were tested } \\
\hline \multirow[b]{2}{*}{ Gene } & \multicolumn{3}{|c|}{ Build 36} & \multicolumn{3}{|c|}{ Build 37} \\
\hline & \multicolumn{2}{|l|}{ Location } & Size $(\mathbf{k b})$ & \multicolumn{2}{|c|}{ Location } & size $(\mathbf{k b})$ \\
\hline EPHB6 & \multicolumn{2}{|c|}{ chr7: $142,252,914-142,288,969$} & 36.06 & \multicolumn{2}{|c|}{$\operatorname{chr} 7: 142,542,792-142,578,847$} & 36.06 \\
\hline EFNB2 & \multicolumn{2}{|c|}{ chr13: $105,930,097-105,995,338$} & 65.24 & \multicolumn{2}{|c|}{ chr 13: 107,132,079-107,197,388 } & 65.31 \\
\hline EFNB3 & \multicolumn{2}{|c|}{ chr17: 7,539,245-7,565,418 } & 26.17 & \multicolumn{2}{|c|}{ chr $17: 7,598,520-7,624,693$} & 26.17 \\
\hline GRIP1 & \multicolumn{2}{|c|}{ chr12: $65,019,066-65,369,020$} & 349.95 & \multicolumn{2}{|c|}{ chr 12: 66,731,211-67,082,925 } & 351.71 \\
\hline \multicolumn{7}{|c|}{ Minimum p-values from IBPC meta-analysis among SNPs examined within EPHB6, EFNB2, EFNB3 and GRIP1 genes } \\
\hline \multirow{2}{*}{ Gene } & \multirow{2}{*}{ Number of SNPs examined } & \multirow{2}{*}{ LD Blocks $\mathbf{r} 2=0.8$} & \multicolumn{2}{|c|}{ Diastolic Pressure } & \multicolumn{2}{|c|}{ Systolic Pressure } \\
\hline & & & SNP & p-value & SNP & p-value \\
\hline EPHB6 & 48 & 4 & rs1009848 & 0.0373 & rs2299557 & 0.404 \\
\hline EFNB2 & 54 & 24 & rs2057408 & 0.077 & rs9520087 & 0.218 \\
\hline EFNB3 & 6 & 6 & rs3744258 & 0.201 & rs3744258 & 0.191 \\
\hline GRIP1 & 420 & 132 & rs1495496 & 0.000389 & rs1495496 & 0.00144 \\
\hline Total & 528 & 166 & \multicolumn{4}{|c|}{ Critical p value: $0.05 / 166=0.0003012$} \\
\hline
\end{tabular}

Table 1. Association of SNPs in the EPHB6/EFNB system with BP phenotypes in 69,396 human subjects.

signaling has a default function of reducing VSMC contractility, leading to lower BP. In the absence of such reverse signaling, such as the case of EPHB6 KO, the VSMC contractility will increase, resulting higher BP.

In support of this notion, we have shown that deletion of EFNB1, a ligand of EPHB6, results in a hypertensive phenotype in mice ${ }^{23}$. Therefore, we identified EPHB6 and, by logical extension and with certain experimental evidence, its ligands (EFNBs) as novel BP regulatory factors in animal models.

In order to establish the relevance of our findings in mice to human blood pressure regulation, we selected 528 single nucleotide polymorphisms (SNPs) within the genes of EPHB6, EFNB2, EFNB3 and glutamate receptor interacting protein 1 (GRIP1), which is a key molecule in EFNB reverse signalling, to query the International Blood Pressure Consortium (IBPC) dataset, which contains SNP information on 69,395 individuals. We found that a SNP in the GRIP1 gene approached statistical significance for association with diastolic blood pressure in humans. Additional animal studies revealed roles of EFNB3 and GRIP1in regulating arterial tone and VSMC contractility, providing phenotypic evidence supporting the genetic findings in humans.

\section{Materials and Methods}

Meta-analysis of SNPs in EPHB and EFNB genes and a related gene GRIP1 for association with BP phenotypes in humans. The $p$-values for association with diastolic pressure (DP) and systolic pressure (SP) were calculated for a total of 528 SNPs found within the regions of 4 genes (EPHB6, EFNB2, EFNB3 and

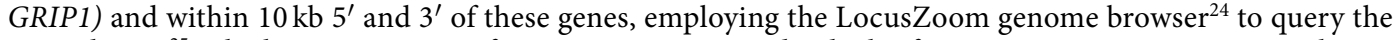
IBPC dataset ${ }^{25}$, which contains SNP information on 69,395 individuals of European ancestry in 29 general population-based cohorts.

These 528 SNPs represented 166 independent linkage disequilibrium (LD) blocks, as determined by the Tagger program ${ }^{26}$ on the HapMap website ${ }^{27}$. Table 1 lists the genes and regions in which the SNPs are located. Query of 166 independent LD blocks resulted in a Bonferroni-corrected critical $p$-value of 0.0003012 for a given $\mathrm{BP}$ phenotype (systolic or diastolic pressure). Looking for the best results between systolic or diastolic pressure phenotypes would require a lower critical $p$-value; however, correcting for $2 \times 166$ tests $(\mathrm{p}=0.000151)$ would be overly conservative as these 2 measurements are not independent.

This human genetic study was carried out in accordance with relevant guide lines of the participating institutions. The research protocol was approved by the institutional ethics committees of the institutions. Informed consent was obtained from all the subjects used in this study.

Efnb3 KO mice. Efnb3 KO mice were produced by Regeneron Pharmaceuticals, as described previously ${ }^{28}$, and generously provided to us. They had been backcrossed to the C57BL/6 background for more than 10 generations. Age- and gender-matched WT littermate mice served as controls and are referred to as WT mice. The mice were housed in ambient temperature $\left(22^{\circ} \mathrm{C}\right)$ with 12 -h light and dark cycles. For all the in vivo experiments, mice were housed one per cage.

Echocardiography. Transthoracic echocardiography was undertaken in mice lightly anesthetized with isoflurane. The experiments were always conduced around noon time. The oestrus cycles of the females were not monitored. Their carotid vessels and heart were imaged with a high-resolution ultrasound biomicroscope (Vevo770; Visualsonics, Toronto, ON, Canada) equipped with a $100 \%$ bandwidth $30-\mathrm{MHz}$ central frequency transducer (RMV-707, $12.7 \mathrm{~mm}$ focal length, $6 \mathrm{~mm}$ aperture). Lateral and axial resolutions with this probe are $\sim 115 \mu \mathrm{m}$ and $\sim 55 \mu \mathrm{m}$, respectively ${ }^{29}$. Preheated ultrasound transmission gel (Aquasonic 100, Parker Laboratories, Orange, NJ, USA) was placed on regions of interest to provide acoustic coupling medium between the transducers and animals. The left and right common carotid arteries were imaged longitudinally in B mode to guide recordings of Doppler time-varying flow velocities for $2 \mathrm{~s}$. Doppler sample volume was positioned 1-2 mm prior to the carotid bifurcation at a $60^{\circ}$ angle. Acquired angle-corrected Doppler data were analyzed to measure the mean Pourcelot index (PI) over 10 consecutive cardiac cycles, according to Stoyanova et al. ${ }^{30}$. The PI is a dimensionless 
echocardiographic parameter that characterizes vascular hemodynamics downstream of a measurement point. It depends on both arterial compliance and downstream vascular resistance.

The heart was also imaged in B mode via the parasternal long-axis view to assess aortic hemodynamics and cardiac output (CO). The M-mode sampling line was positioned perpendicularly to the ascending aorta, 0.5-1.5 mm downstream of the aortic valve, and time-varying tracings tracked changes in aortic diameter (AoD). Mean AoD was assessed over 5 consecutive cardiac cycles. Doppler velocity waveforms were then recorded in the ascending aorta by positioning sampling volume at the exact same location where $\mathrm{M}$-mode tracings were obtained. The envelope of angle-corrected $\left(60^{\circ}\right)$ Doppler tracings was delineated manually to compute the velocity time integral (VTI), which was averaged over 10 cardiac cycles. Assuming a parabolic velocity profile in the ascending aorta ${ }^{30}$, stroke volume (SV) was calculated (in $\left.\mathrm{ml}\right)$ as $1 / 2(\mathrm{AoD} / 2)^{2} \times \pi \times \mathrm{VTI}$, and CO (in $\mathrm{ml} / \mathrm{min}$ ) was estimated as $\mathrm{SV} \times$ heart rate $(\mathrm{HR})$, where HR was mean HR of the animals.

Left ventricle (LV) dimensions (in $\mathrm{mm}$ ) at end-systole and end-diastole were finally assessed to quantify fractional shortening, ejection fraction and LV mass. B-mode parasternal long-axis viewing guided the capture of M-mode tracings through the anterior and posterior LV walls at the level of the papillary muscle. For each mouse, LV end-diastolic diameter (LVEDD), LV end-systolic diameter (LVESD), LV end-diastolic posterior wall thickness (LVEDPW) and intra-ventricular septum dimension at end-diastole (IVSED) were quantified, and LV mass ascertained in $\mathrm{mg}$ with the following equation ${ }^{31,32}$ :

$$
\text { LV mass }(\mathrm{mg})=1.055 \times[(\text { LVEDD }+ \text { LVEDPW }+ \text { IVSED })-\text { LVEDD }]
$$

A visual illustration of methods of mouse cardioechography can be found in a video publication by Respress and Wehren ${ }^{33}$.

VSMC isolation. Mouse VSMC were isolated from aortic and mesenteric arteries, including their secondary branches as described before ${ }^{22,23}$.

siRNA transfection and reverse transcription-quantitative polymerase chain reaction (RT-qPCR). The sequences of Grip1, Pdg-rgs3, and Disheveled siRNA and siRNA transfection procedures are described in our previous publication ${ }^{22}$. Their mRNA levels in VSMCs were measured by RT-qPCR; the primer sequences and qPCR cycling program are detailed before ${ }^{22} . \beta$-actin mRNA served as internal control. Samples were tested in triplicate, and the data were expressed as signal ratios of test gene mRNA/ $\alpha$-actin mRNA.

VSMC contractility. VSMC contractility was measured as described before ${ }^{22,23}$. Briefly, the cells were cultured in wells coated with goat anti-mouse EFNB3 Ab (sc-7281, Santa Cruz Biotechnology, Dallas, Texas) or control goat IgG $(2 \mu \mathrm{g} / \mathrm{ml}$ during coating). After 3-4 days, they were stimulated with PE $(20 \mu \mathrm{mol} / \mathrm{L})$, and photographed continuously for $15 \mathrm{~min}$ at a rate of 1 picture per min. Fifteen or more cells were randomly selected, and their length was measured at each time point with Zeiss Axiovision software. Percentage contraction was calculated as follows:

$$
\% \text { contraction }=100 \times(\text { cell length at time } 0-\text { cell length at time } \mathrm{X}) / \text { cell length at time } 0 .
$$

Ethics Statement. This study was carried out in strict accordance with the recommendations in the Guide for the Care and Use of Laboratory Animals of the National Institutes of Health. The protocol was approved by the Institutional Committee on Animal Care of CRCHUM (Permit Number: 4I14033JWs). All surgery was performed under isoflurane anesthesia, and all efforts were made to minimize suffering. Informed consent was obtained from all the subjects used in this study.

\section{Results}

Association of SNPs in the EPHB/EFNB system with BP phenotypes. Our previous studies ${ }^{22,23}$ along with some of our unpublished observations indicate that molecules in the EPHB and EFNB families (e.g., EPHB6, EFNB1, EFNB2, and EFNB3) and a certain adaptor protein (GRIP1) within their signalling pathways are novel factors that can modulate BP in mice. The relevance of these molecules in human BP regulation was investigated. The IBPC conducted a meta-analysis of genome-wide association scanning (GWAS) in 69,395 individuals of European ancestry in 29 cohorts from European and North American countries ${ }^{25}$. Two and a half million genotyped or imputed SNPs were tested for their association with SP and DP in these individuals. We queried the results of this meta-analysis for association of 528 SNPs in EPHB6, EFNB2, EFNB3 and GRIP1 genes with systolic or diastolic pressure in these individuals. EFNB1 was not included in the analysis because it is an X-linked gene, and its SNP information is not available in the IBPC dataset. The $p$-values of these SNPs for their association with systolic or diastolic pressure are illustrated in Supplementary Figure 1 (S. Fig. 1). Table 1 summarizes the names of SNPs with the most significant association and their $p$-values. The minimum observed $p$-value for any association (0.000389) was for the association of SNP rs1495496 located within the GRIP1 gene with diastolic pressure. This value approaches the critical $p$-value of 0.000302. For the other 3 genes analyzed, i.e., EPHB6, EFNB2 and EFNB3, the minimum $p$-values of their SNPs did not approach the critical $p$-value. The implications of these findings are elaborated in the Discussion.

Small artery resistance in EFNB3 KO mice in vivo. EFNB3 is an EPHB6 ligand, and its function might contribute to the BP phenotype observed in Ephb6 $\mathrm{KO}$ mice $^{22}$. We assessed several BP related parameters in Efnb3 $\mathrm{KO}$ mice (called KO mice hereafter) using echocardiography. 


\begin{tabular}{|c|c|c|c|c|c|}
\hline Mouse Type & Age & Cardiac output (ml/min) & Left Carotid PI & Right Carotid PI & LV Mass (mg) \\
\hline \multicolumn{6}{|c|}{ Echocardiographic parameters of male Efnb3 KO mice } \\
\hline KO male & $15 \mathrm{wk}$ & 14.65 & 0.75 & 0.72 & 103.7 \\
\hline KO male & $15 \mathrm{wk}$ & 21.46 & 0.87 & 0.68 & 125.8 \\
\hline KO male & $15 \mathrm{wk}$ & 24.79 & 0.73 & 0.78 & 154.1 \\
\hline KO male & $15 w k$ & 17.23 & 0.74 & 0.70 & 91.7 \\
\hline KO male & $15 \mathrm{wk}$ & 14.93 & 0.78 & 0.78 & 89.7 \\
\hline KO male & $14 \mathrm{wk}$ & 18.2 & 0.73 & 0.72 & 133.6 \\
\hline KO mean & & 18.54 & 0.77 & 0.73 & 116.4 \\
\hline SD & & 3.939 & 0.0533 & 0.041 & 25.65 \\
\hline WT male & $14 \mathrm{wk}$ & 21.5 & 0.83 & 0.84 & 116.0 \\
\hline WT male & $15 \mathrm{wk}$ & 15.33 & 0.74 & 0.70 & 140.5 \\
\hline WT male & $15 \mathrm{wk}$ & 23.52 & 0.75 & 0.77 & 115.6 \\
\hline WT male & $15 \mathrm{wk}$ & 18.21 & 0.73 & 0.72 & 133.6 \\
\hline WT male & $14 \mathrm{wk}$ & 31.85 & 0.77 & 0.86 & 115.0 \\
\hline WT male & $14 \mathrm{wk}$ & 28.57 & 0.77 & 0.81 & 145.1 \\
\hline WT male & $14 \mathrm{wk}$ & 24.19 & 0.74 & 0.76 & 152.7 \\
\hline WT male & $14 \mathrm{wk}$ & 30.95 & 0.79 & 0.83 & 175.6 \\
\hline WT mean & & 24.265 & 0.77 & 0.78 & 126.4 \\
\hline SD & & 5.92 & 0.030 & 0.047 & 12.57 \\
\hline$p$ value (t test) & & 0.0637 & 0.9075 & 0.08200 & 0.4967 \\
\hline \multicolumn{6}{|c|}{ Echocardiographic parameters of female Efnb3 KO mice } \\
\hline KO female & $15 \mathrm{wk}$ & 21.89 & 0.75 & 0.78 & 112.3 \\
\hline KO female & $15 \mathrm{wk}$ & 16.29 & 0.77 & 0.8 & 92.5 \\
\hline KO female & $14 \mathrm{wk}$ & 20.16 & 0.81 & 0.81 & 121.1 \\
\hline KO female & $15 \mathrm{wk}$ & 27.57 & 0.76 & 0.77 & 143.3 \\
\hline KO female & $15 \mathrm{wk}$ & 20.54 & 0.94 & 0.76 & 130.5 \\
\hline KO female & $14 \mathrm{wk}$ & 27.71 & 0.75 & 0.73 & 134.3 \\
\hline KO female & $15 \mathrm{wk}$ & 19.13 & 0.8 & 0.77 & 135.8 \\
\hline KO female & $14 w k$ & 18.38 & 0.81 & 0.82 & 122 \\
\hline KO mean & & 21.45 & 0.79 & 0.78 & 123.9 \\
\hline SD & & 4.153 & 0.062 & 0.029 & 16.04 \\
\hline WT female & $14 \mathrm{wk}$ & 33.49 & 0.72 & 0.72 & 109 \\
\hline WT female & $14 \mathrm{wk}$ & 18.92 & 0.69 & 0.67 & 118.1 \\
\hline WT female & $14 \mathrm{wk}$ & 29.03 & 0.71 & 0.8 & 110.9 \\
\hline WT female & $14 \mathrm{wk}$ & 28.06 & 0.81 & 0.79 & 95.9 \\
\hline WT female & $14 \mathrm{wk}$ & 15.96 & 0.68 & 0.76 & 114.6 \\
\hline WT female & $14 \mathrm{wk}$ & 22.46 & 0.7 & 0.74 & 72.2 \\
\hline WT mean & & 24.65 & 0.71 & 0.74 & 93.4 \\
\hline SD & & 6.664 & 0.047 & 0.048 & 29.98 \\
\hline p value (t test) & & 0.289 & 0.0218 & 0.132 & 0.0397 \\
\hline
\end{tabular}

Table 2. Echocardiographic analysis of $\mathrm{CO}$, carotid artery resistance and left ventricle mass of Efnb3 KO and WT mice. BP-related echocardiographic parameters of individual Efnb3 KO and WT mice are reported. Means \pm SD are shown at the end of each group, and $p$ values (unpaired one-way Student's $t$ test) are indicated at the bottom of Tables 2. PI: Pourcelot index; LV: left ventricle.

$\mathrm{BP}$ is a function of CO, blood volume and blood vessel flow resistance. Echocardiography was employed to examine CO, the PI (a parameter reflecting blood vessel flow resistance) of carotid arteries, and LV mass in live KO and WT mice. Due to technical limitations, we were not able to conduct echocardiography in smaller arteries. CO in male (Table 2) and female (Table 2) KO mice was comparable to that in WT controls, indicating that this parameter, which could affect BP, is not under the influence of EFNB3. However, the left carotid PI in female KO (Table 2) but not male KO (Table 2) mice was significantly higher than that of WT counterparts $(\mathrm{p}=0.0218)$, reflecting heightened resistance of these small arteries. LV mass increased significantly in female but not in male KO mice, compared to their WT counterparts $(\mathrm{p}=0.0397$, Table 2$)$. This hypertrophy might be the result of augmented cardiac workload in female KO mice to overcome the heightened blood flow resistance of their small arteries.

GRIP1 knockdown by siRNA cancelled the effect of solid phase anti-EFNB3 Ab on the contractility of WTVSMCs. The echocardiography results suggest that the default function of EFNB3 is vessel relaxation, and hence the EFNB3 KO carotid artery manifested increased resistance. To prove this at the cellular level, we triggered EFNB3 reverse signalling by incubating WT VSMCs in wells coated with anti-EFNB3 Ab and tested 
A
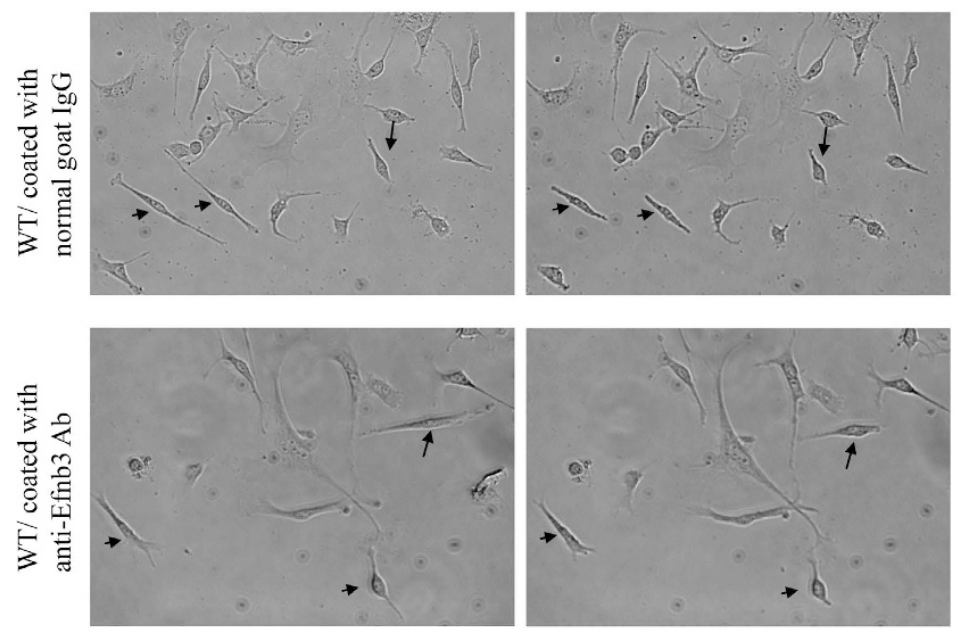

$\mathrm{B}$

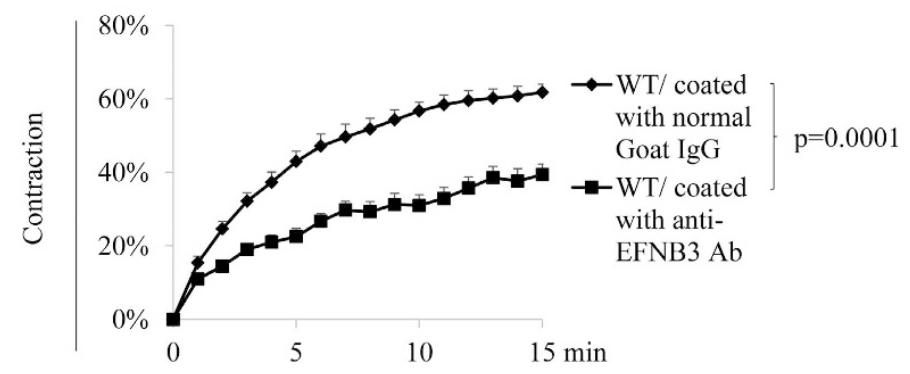

Figure 1. Crosslinking EFNB3 on VSMCs results in decreased contractility. VSMCs from female WT mice were cultured in wells coated with goat anti-mouse EFNB3 Ab or control goat IgG $(2 \mu \mathrm{g} / \mathrm{ml}$ during coating) for 4 days. The cells were then stimulated with PE $(20 \mu \mathrm{mol} / \mathrm{L})$, and their percentage contraction was recorded by microscopy. (A) Micrographs showing the contraction of WT VSMCs in the presence or absence of solid phase anti-EFNB3. Upper row: WT VSMCs cultured in wells coated with control goat IgG (20 $\mu \mathrm{g} / \mathrm{ml}$ for coating). Lower row: WT VSMCs cultured in wells coated with goat anti-mouse EFNB3 Ab. The images were taken at 0,7 and 15 min after PE stimulation. Arrows mark the same cells in each row at different time points, to show the shortening of the cells. (B) Reduced contractility of WT VSMCs cultured in the presence of solid phase anti-EFNB3 Ab. VSMCs from female WT mice were cultured in wells coated with normal goat IgG or goat anti-mouse EFNB3 Ab for 4 days. The cells were then stimulated with PE, and were imaged at one frame per $\min$ for $15 \mathrm{~min}$. Means $\pm \mathrm{SD}$ of the percentage contraction during a 15 -min period are reported. The percentage contraction is calculated as follows. \% contraction = length of cells at a given timepoint/length of the cells at time 0 . The data were analyzed with one-way ANOVA, and $p$-value is indicated. The experiment in this figure was repeated three times, and representative data are shown.

their contractility. Such solid phase anti-EFNB3 Ab serves as an agonist to EFNB3, because it acts at the bottom of the cells and will not block putative EFNB3/EPH interaction which might exist at the vertical side of neighboring VSMCs. Further, during to the low density used in culture, such fraternal cell interaction is rare. A similar strategy of solid phase anti-EFNB1 Ab as an agonist to trigger EFNB1 reverse signalling in VSMCs has been previously published $^{23}$. As shown in Fig. 1A and B, WT VSMCs crosslinked with anti-EFNB3 Ab presented significantly reduced contractility, compared to those cultured in wells coated with normal goat IgG. On the other hand, KO VSMC contractility was not affected by anti-EFNB3 crosslinking, as expected, because they have no EFNB3 expression (data not shown). These results corroborate that of echocardiography and confirm that EFNB3 reverse signalling leads to reduced VSMC contractility. The solid phase anti-EFNB3 Ab indeed triggered signalling in VSMCs in that their ERK phosphorylation upon PE stimulation was increased (S. Fig. 2).

EFNBs had no enzymatic activity. However, EFNBs engage adaptor proteins to link their intracellular tails to various signalling pathways. Grip1 siRNA was used to knock down the expression of this adaptor protein in the WT and KO VSMCs, as it is known to associate with EFNBs ${ }^{34-36}$. The effectiveness of mRNA knockdown was verified by RT-qPCR (Fig. 2A). EFNB3 expression in the WT VSMCs was not affected by the knockdown (S. Fig. 3). WT VSMCs were cultured in wells coated with anti-EFNB3 Ab to invoke EFNB3 reverse signalling, and KO VSMCs were used as controls. In WT VSMCs, such treatment (Fig. 2B) dampened VSMC contractility of WT VSMCs transfected with control siRNA, but such dampening effect was reversed by Grip1 siRNA (Fig. 2B). KO VSMCs cultured in wells coated with anti-EFNB3 Abs had a higher contraction, as they had no EFNB3 and were not affected by anti-EFNB3-evoked reverse signalling, which could reduce contractility. As expected, Grip1 siRNA knockdown did not affect KO VSMC contractility, because GRIP1 was not utilized by the KO VSMCs. The effect of GRIP1 knockdown on the augmentation of WT VSMC contractility depended on the existence of EFNB3 
A

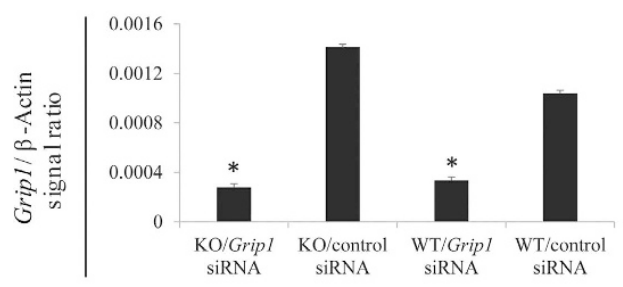

B

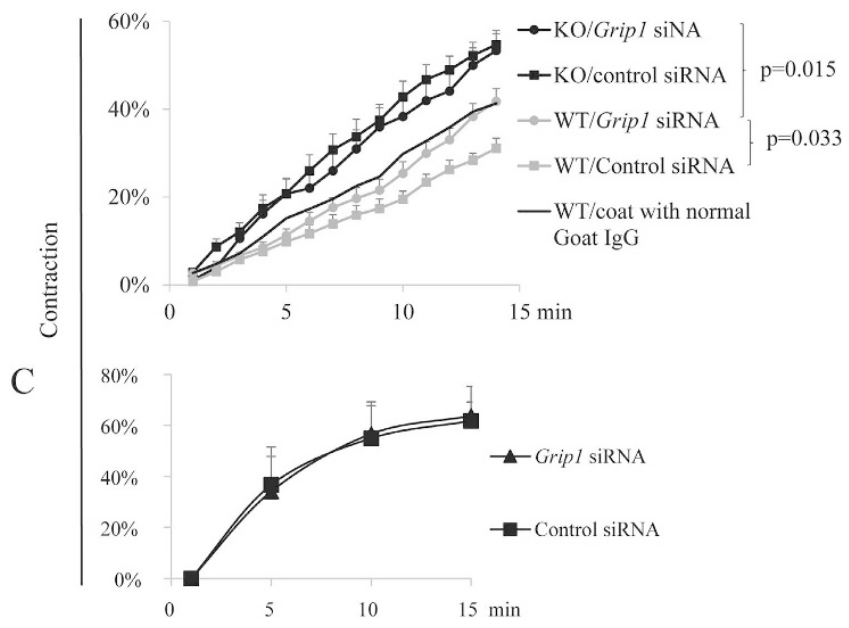

Figure 2. GRIP1 in the EFNB3 reverse signaling pathway in VSMCs. Experiments in this figure were repeated more than twice, and representative data are shown. (A) Effective mRNA knockdown of Grip1 by siRNA. VSMCs from female WT mice were transfected with siRNAs of a particular gene or control siRNA, as indicated. After 24-h culture, the cells were harvested and the mRNA expression of each gene was determined by RT-qPCR. The data are expressed as means \pm SD of the ratios of the target gene signal versus the $\beta$-actin signal. The data were analyzed by one-way Student's $t$ test. ${ }^{*} \mathrm{p}<0.05$. (B) GRIP1 knockdown by siRNAs reverses the effect of solid-phase anti-EFNB3 Ab on reducing VSMC contractility. VSMCs from female WT and KO mice were cultured in wells coated with goat anti-mouse EFNB3 Ab ( $2 \mu \mathrm{g} / \mathrm{ml}$ during coating). After 2 days, they were transfected with siRNAs targeting Grip1, or with control siRNA. On day 4 of culture, they were stimulated with PE $(20 \mu \mathrm{mol} / \mathrm{L})$, and their percentage contraction was recorded. Means \pm SD of the percentage are reported. The thin line represents mean contractility of VSMCs cultured in well without coating of anti-EFNB3 $\mathrm{Ab}$ as an additional control; for better viewing, $\mathrm{SD}$ was not added to the line. The data were analyzed with oneway ANOVA followed by ad hoc analysis, and p-values of significant difference are indicated. (C) Grip1 siRNA in the absence of EFNB3 reversing signaling had no effect on VSMC contractility. VSMCs from female WT mice were cultured in plain wells without Ab coating. They were transfected with Grip1 or control siRNA and then stimulated with PE as described in $(\mathbf{A})$. Mean \pm SD of percentage contraction are shown. The data were analyzed with one-way ANOVA but not statistically significant difference between the test and control groups is found.

reverse signalling, as Grip1 siRNA had no effect on the contractility of WT VSMCs cultured in wells without anti-EFNB3 Ab coating (Fig. 2C). DISHEVELLED and PDZ-RGS3 are two other proteins capable of associating with the EFNB3 intracellular tail ${ }^{35,36}$. However, their knockdown by siRNA had no effect on WT VSMC contractility (Fig. 3), suggesting that they were not involved in EFNB3 reverse signalling in VSMCs.

These results corroborate the finding of the human genetic study, indicating that GRIP1 is involved in EFNB3 reverse signalling and VSMC contractility regulation, and it is hence a factor modulating blood pressure.

\section{Discussion}

EPHs and EFNs perform critical functions in many tissues and organs. In our previous studies, we found that Ephb6 KO and Efnb1 $\mathrm{KO}$ mice have augmented $\mathrm{BP}^{22,23}$. In the current investigation, we provided additional human genetic evidence showing that the mutations in GRIP1, a molecule in the EPHB6/EFNB signalling pathway and an adaptor protein capable of binding to EFNBs, were associated with human blood pressure phenotype. We also provided mouse experimental data showing that the default function of EFNB3, one of the ligands of EPHB6, was to reduce arterial tone, and such function depended on signalling via GRIP1, corroborating the human genetic findings.

As of today, no genes in the EPHB/EFNB family have been identified as hypertension risk genes in several large-scale GWAS $25,37-44$. There are a couple of possible reasons. 1) The contribution of genes in the EPHB/EFNB family to the BP phenotype might be relatively small, and the possible association is rendered undetectable due to heavy statistical penalties of multiple testing in GWAS. 2) In order to reduce the effect of type 2 error resulting 
A
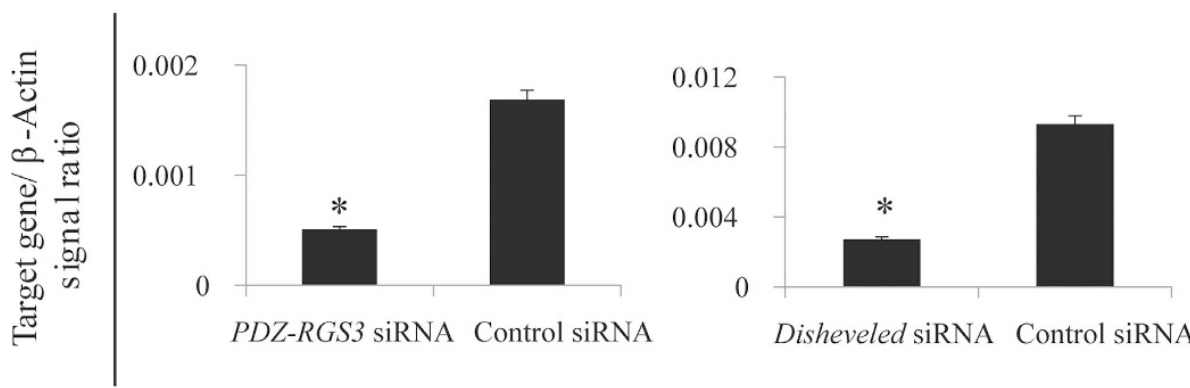

$\mathrm{B}$
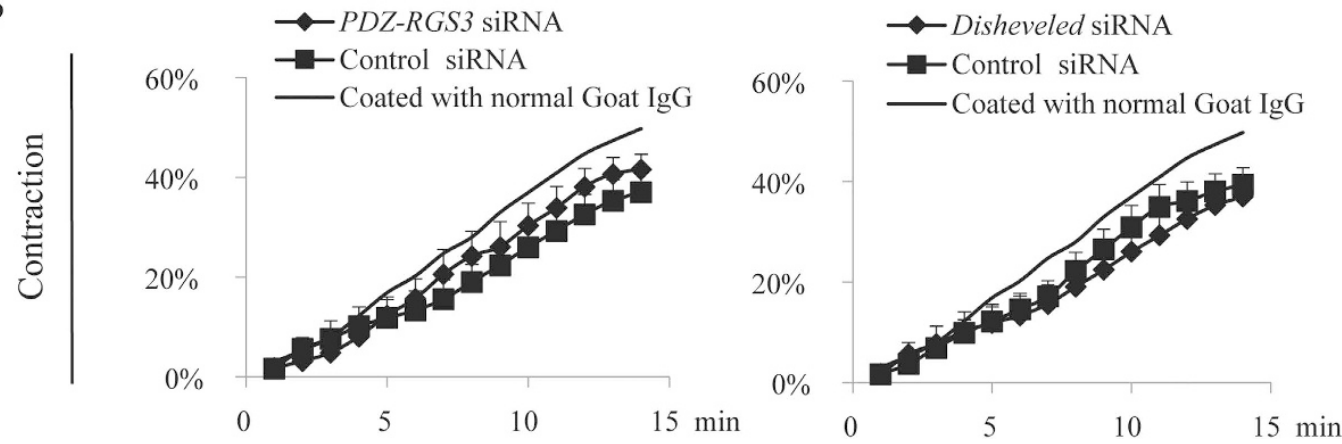

Figure 3. DISHEVELLED and PDZ-RGS3 are not in the EFNB3 reverse signaling pathway in VSMCs. Experiments in this figure were repeated more than twice, and representative data are shown. (A) Effective mRNA knockdown of Disheveled and PDZ-RGS3 by siRNA. VSMCs from female WT mice were transfected with siRNAs of Disheveled and PDZ-RGS3 as described in Fig. 2. The mRNA expression of each gene was determined by RT-qPCR. The data are expressed as means \pm SD of the ratios of the target gene signal versus the $\beta$-actin signal. The data were analyzed by Student's $t$ test. ${ }^{*} p<0.05$. (B) Dishevelled and PDZ-RGS3 knockdown by siRNAs had no effect on WT VSMC contractility. Contractility of VSMCs from female WT mice were assessed in the presence of EFNB3 reverse signalling and DISHEVELLED/PDZ-RGS3 knockdown, as described in Fig. 2B. The data were analyzed with one-way ANOVA but no significant difference was observed.

from multiple-testing correction in genome-wide association studies, the IBPC study has assembled very large samples by combining cohorts from many sub-studies. The hypothesis is that increasing sample size will increase power, allowing the decrease in both type 1 and type 2 errors. However, $p$-value is controlled by both sample size and effect size (odds ratio) and, of the two, a slight decrease in effect size has a more dramatic impact on $p$-value. Assembly of large disparate cohorts does increase sample size but it also introduces increased heterogeneity, both phenotypic and genetic. Such heterogeneity may reduce the average effect size.

We conducted a more focused query of the IBPC dataset, using only 4 genes in the EPHB/EFNB signalling pathway, and discovered that a SNP, rs1495496, located between exons 22 and 23 of GRIP1, had a $p$-value of 0.000389 for its association with DP, which approaches the Bonferroni corrected critical $p$-value 0.000302 . This finding is very promising since Bonferroni correction by itself was deemed to be quite conservative. Considering our observation that EPHBs/EFNBs influence vessel tone in mice, the possible association of this SNP in GRIP1 gene with DP seems logical. Why does only a SNP from GRIP1 approach significance and not those from 3 EPHB6/EFNBs queried? GRIP1 is situated in a node of EPHB6, EFNB1 and EFNB3 reverse signalling pathway; it thus probably carries more weight than each individual EPHB or EFNB member and, hence, is easier to detect for its associations with BP phenotypes. Our recent published results ${ }^{22,45}$ and unpublished observations in animal models indicate that the effect of EPHBs and EFNBs on blood pressure regulation is not only sex-dependent, but also sex hormone level-dependent. It is possible that in a cohort stratified by sex and sex hormone levels, more significant association of BP phenotypes with SNPs from GRIP1 and EPHB/EFNB family members will be detected in humans, and this will fully establish the relevance of our findings from animal models to human blood regulation.

Our cardioechogrphy data using EFNB3 KO mice suggest a default sex/sex hormone-dependent vasolaxative role of EFNB3 in that its deletion led to increased blood flow resistance in the carotid artery of female KO but not male KO mice, compared to their WT counterparts. Such increased resistance is not due to developmental changes of the arteries in the KO mice, as we found no abnormalities in the aorta and mesenteric arteries in terms of histology and diameters (S. Fig. 4).

Using telemetry, we have recently revealed that female but not male EFNB3 KO mice have increased BP, but after ovariectomy, the female KO BP returns to the normal range ${ }^{46}$. This finding corroborates the data from cardioechography, suggesting that after EFNB3 deletion, increased vascular tone results in increased BP, but such effect is sex- and sex hormone level-dependent. 
We demonstrated EFNB3 can modulate VSMC contractility by reverse signalling. EFNB3 is a transmembrane protein without enzymatic activity in its intracellular tail. How does it regulate VSMC contractility? We have demonstrated that the association of EFNB3 with GRIP1 is critical for its function in regulating VSMC contractility. In VSMCs, EFNB3 might also associate with other so-far unidentified binding proteins. During reverse signaling, some of these proteins might interact with and modulate the functions of other pathways that control VSMC contractility, the MLCK pathway being one of them. The intermediate signalling molecules between EFNB3/ GRIP1 and MLCK/MLC remain to be identified.

In our previous publication, we reported that deletion of EPHB4 in mice results in reduced BP, and GRIP1 is involved in the signaling between EPHB4 and its EFN ligands ${ }^{45}$, most likely its major ligand EFNB2. In this study, we showed that GRIP1 is also involved in EFNB3 signaling, the deletion of which leads to increased BP. How do we explain that the same molecule is mediating signals that resulting opposite phenotype in the same type of VSMCs? In the biological system, it is not uncommon that a molecule can mediate multiple different functions, depending to the status of a cell and the different upstream signals it receives. For example, $\beta$-catenin can mediate many different cellular events, such as cell adhesion, stem cell renewal, asymmetric cell division, etc., depending which binding partners it associates with ${ }^{46}$. GRIP1 is no different in this sense: it can mediate signaling leading to increased or decreased VSMC contraction, depending on its upstream signaling: from EFNB (the preferred ligand of EPHB4) or EFNB3, respectively.

What is the possible mechanism by which estrogen in concert with EFNB3 modulates VSMC contractility? We have demonstrated that estrogen augments KO but not WT VSMC contractility, and this is mediated by its nongenomic effect via cell surface receptor GPR30. GRP30 can directly regulate ERK activity ${ }^{47}$, which in turn controls VSMCs contractility ${ }^{48}$. As EFNB3 reverse signalling can activate ERK as shown in S. Fig. 2, ERK or molecules in this category which modulates VSMC activity both in the presence or absence of EFNB3, can function as a node on which EFNB3 and GPR30 signalling pathways converge. This could be a possible mechanism by which EFNB3 and estrogen act in concert in regulating VSMC contractility. Our additional study revealed that EFNB3 deletion upregulates GPR30 expression and the upregulation is at the transcription level ${ }^{49}$. There was no evidence of direct interaction between EFNB3 and GPR30, according to fluorescent resonance energy transfer assays (data not shown).

Our research on the roles of EPHBs/EFNBs in VSMC contractility has revealed a group of previously unknown molecules capable of regulating vascular tone and blood pressure. Among this group of molecules, we have shown that the default function of EPHB6, EFNB1 and EFNB3 is to reduce vascular tone ${ }^{22,23,49}$, and thus their deletion leads to increased vessel contractility and blood pressure. Our most recent finding indicates that among the EPHBs and EFNBs, there are another group of molecules such as EPHB4 and its major ligand EFNB2, whose default function is opposite, i.e., to enhance the vascular tone ${ }^{45,50}$; consequently, their deletion lead to reduced blood pressure. These opposing forces of different member of EPHBs and EFNBs are like Yin and Yang and probably play a role for the fine tuning of the vascular tone. A better understanding of the physiological relevance and mechanisms of action of these molecules for their role in vascular contractility and blood pressure regulation will afford us a novel personalized therapeutic approach to blood pressure management. For example, female hypertensive patients with EFNB3 mutations could be identified by genetic testing, and for this subpopulation of patients, avoidance of oral contraceptive/hormone replacement could reduce their hypertension risks.

\section{References}

1. Unified nomenclature for Eph family receptors and their ligands, the ephrins. Eph Nomenclature Committee. Cell 90, 403-404, doi: 10.1016/S0092-8674(00)80500-0 (1997).

2. Flanagan, J. G. \& Vanderhaeghen, P. The ephrins and Eph receptors in neural development. Annu Rev Neurosci 21, 309-345, doi: 10.1146/annurev.neuro.21.1.309 (1998).

3. Pasquale, E. B. Eph-ephrin bidirectional signaling in physiology and disease. Cell 133, 38-52, doi: 10.1016/j.cell.2008.03.011 (2008).

4. Wilkinson, D. G. Eph receptors and ephrins: regulators of guidance and assembly. Int Rev Cytol 196, 177-244, doi: 10.1016/S00747696(00)96005-4 (2000).

5. Luo, H., Wan, X., Wu, Y. \& Wu, J. Cross-linking of EphB6 resulting in signal transduction and apoptosis in Jurkat cells. J. Immunol 167, 1362-1370, doi: 10.4049/jimmunol.167.3.1362 (2001).

6. Luo, H., Yu, G., Wu, Y. \& Wu, J. EphB6 crosslinking results in costimulation of T cells. J. Clin. Invest 110, 1141-1150, doi: 10.1172/ JCI15883 (2002).

7. Yu, G., Luo, H., Wu, Y. \& Wu, J. Ephrin B2 induces T cell costimulation. J. Immunol 171, 106-114, doi: 10.4049/jimmunol.171.1.106 (2003).

8. Yu, G., Luo, H., Wu, Y. \& Wu, J. Mouse ephrinB3 augments T-cell signaling and responses to T-cell receptor ligation. J. Biol. Chem 278, 47209-47216, doi: 10.1074/jbc.M306659200 (2003).

9. Luo, H., Yu, G., Tremblay, J. \& Wu, J. EphB6-null mutation results in compromised T cell function. J. Clin. Invest 114, 1762-1773, doi: 10.1172/JCI21846 (2004).

10. Yu, G., Luo, H., Wu, Y. \& Wu, J. EphrinB1 is essential in T-cell-T-cell co-operation during T-cell activation. J. Biol. Chem 279, 55531-55539, doi: 10.1074/jbc.M410814200 (2004).

11. Wu, J. \& Luo, H. Recent advances on T-cell regulation by receptor tyrosine kinases. Curr Opin Hematol 12, 292-297, doi: 10.1097/01. moh.0000166497.26397.9f (2005).

12. Yu, G., Mao, J., Wu, Y., Luo, H. \& Wu, J. Ephrin-B1 is critical in T-cell development. J. Biol. Chem 281, 10222-10229, doi: 10.1074/ jbc.M510320200 (2006).

13. Luo, H. et al. Efnb1 and Efnb2 proteins regulate thymocyte development, peripheral T cell differentiation, and antiviral immune responses and are essential for interleukin-6 (IL-6) signaling. J. Biol. Chem 286, 41135-41152, doi: 10.1074/jbc.M111.302596 (2011).

14. Luo, H. et al. Ephrinb1 and Ephrinb2 are associated with interleukin-7 receptor alpha and retard its internalization from the cell surface. J. Biol. Chem 286, 44976-44987, doi: 10.1074/jbc.M111.316414 (2011).

15. Batlle, E. et al. Beta-catenin and TCF mediate cell positioning in the intestinal epithelium by controlling the expression of EphB/ ephrinB. Cell 111, 251-263, doi: 10.1016/S0092-8674(02)01015-2 (2002).

16. Davy, A., Bush, J. O. \& Soriano, P. Inhibition of gap junction communication at ectopic Eph/ephrin boundaries underlies craniofrontonasal syndrome. PLoS Biol 4, e315, doi: 10.1371/journal.pbio.0040315 (2006). 
17. Zhao, C. et al. Bidirectional ephrinB2-EphB4 signaling controls bone homeostasis. Cell Metab 4, 111-121, doi: 10.1016/j. cmet.2006.05.012 (2006).

18. Wang, H. U., Chen, Z. F. \& Anderson, D. J. Molecular distinction and angiogenic interaction between embryonic arteries and veins revealed by ephrin-B2 and its receptor Eph-B4. Cell 93, 741-753, doi: 10.1016/S0092-8674(00)81436-1 (1998).

19. Konstantinova, I. et al. EphA-Ephrin-A-mediated beta cell communication regulates insulin secretion from pancreatic islets. Cell 129, 359-370, doi: 10.1016/j.cell.2007.02.044 (2007)

20. Hashimoto, T. et al. Ephrin-B1 localizes at the slit diaphragm of the glomerular podocyte. Kidney Int 72, 954-964, doi: 10.1038/ si.ki.5002454 (2007).

21. Dravis, C. et al. EphB2 and ephrin-B2 regulate the ionic homeostasis of vestibular endolymph. Hear Res 223, 93-104, doi: 10.1016/j. heares.2006.10.007 (2007).

22. Luo, H. et al. Receptor tyrosine kinase Ephb6 regulates vascular smooth muscle contractility and modulates blood pressure in concert with sex hormones. The J. Biol. Chem 287, 6819-6829, doi: 10.1074/jbc.M111.293365 (2012).

23. Wu, Z. et al. Possible role of Efnb1 protein, a ligand of Eph receptor tyrosine kinases, in modulating blood pressure. J. Biol. Chem 287, 15557-15569, doi: 10.1074/jbc.M112.340869 (2012).

24. University of Michigan, LocusZoom - Create Plots of Genetic Data. http://locuszoom.sph.umich.edu/locuszoom (Date of access:04/2015)

25. International Consortium for Blood Pressure Genome-Wide Association, S. et al. Genetic variants in novel pathways influence blood pressure and cardiovascular disease risk. Nature 478, 103-109, doi: 10.1038/nature10405 (2011).

26. de Bakker, P. I. et al. Efficiency and power in genetic association studies. Nat. Genet 37, 1217-1223, doi: 10.1038/ng1669 (2005)

27. U.S. National Library of Medicine, NCBI retiring HapMap Resource. http://hapmap.ncbi.nlm.nih.gov (Date of access:20/01/2016).

28. Kullander, K. et al. Ephrin-B3 is the midline barrier that prevents corticospinal tract axons from recrossing, allowing for unilateral motor control. Genes Dev 15, 877-888, doi: 10.1101/gad.868901 (2001).

29. Zhou, Y. Q. et al. Comprehensive transthoracic cardiac imaging in mice using ultrasound biomicroscopy with anatomical confirmation by magnetic resonance imaging. Physiol Genomics 18, 232-244, doi: 10.1152/physiolgenomics.00026.2004 (2004).

30. Stoyanova, E., Trudel, M., Felfly, H., Garcia, D. \& Cloutier, G. Characterization of circulatory disorders in beta-thalassemic mice by noninvasive ultrasound biomicroscopy. Physiol Genomics 29, 84-90, doi: 10.1152/physiolgenomics.00305.2005 (2007).

31. Yang, X. P. et al. Echocardiographic assessment of cardiac function in conscious and anesthetized mice. Am J Physiol 277, H1967-1974, doi: http://ajpheart.physiology.org/0363-6135/99 (1999).

32. Pollick, C., Hale, S. L. \& Kloner, R. A. Echocardiographic and cardiac Doppler assessment of mice. J Am Soc Echocardiogr 8, 602-610, doi: 10.1016/S0894-7317(05)80373-6 (1995).

33. Respress, J. L. \& Wehrens, X. H. Transthoracic echocardiography in mice. J. Vis. Exp: JoVE, doi: 10.3791/1738 (2010).

34. Bruckner, K. et al. EphrinB ligands recruit GRIP family PDZ adaptor proteins into raft membrane microdomains. Neuron 22, 511-524, doi: 10.1016/S0896-6273(00)80706-0 (1999).

35. Tanaka, M., Kamo, T., Ota, S. \& Sugimura, H. Association of Dishevelled with Eph tyrosine kinase receptor and ephrin mediates cell repulsion. Embo J 22, 847-858, doi: 10.1093/emboj/cdg088 (2003).

36. Lu, Q., Sun, E. E., Klein, R. S. \& Flanagan, J. G. Ephrin-B reverse signaling is mediated by a novel PDZ-RGS protein and selectively inhibits G protein-coupled chemoattraction. Cell 105, 69-79, doi: 10.1016/S0092-8674(01)00297-5 (2001).

37. Adeyemo, A. et al. A genome-wide association study of hypertension and blood pressure in African Americans. PLoS Genet 5, e1000564, doi: 10.1371/journal.pgen.1000564 (2009).

38. Guo, Y. et al. A genome-wide linkage and association scan reveals novel loci for hypertension and blood pressure traits. PloS one 7, e31489, doi: 10.1371/journal.pone.0031489 (2012).

39. Hiura, Y. et al. A genome-wide association study of hypertension-related phenotypes in a Japanese population. Circ J 74, 2353-2359, doi: 10.1253/circj.CJ-10-0353 (2010).

40. Levy, D. et al. Genome-wide association study of blood pressure and hypertension. Nature genetics 41, 677-687, doi: 10.1038/ng.384 (2009).

41. Org, E. et al. Genome-wide scan identifies $\mathrm{CDH} 13$ as a novel susceptibility locus contributing to blood pressure determination in two European populations. Hum Mol Genet 18, 2288-2296, doi: 10.1093/hmg/ddp135 (2009).

42. Padmanabhan, S. et al. Genome-wide association study of blood pressure extremes identifies variant near UMOD associated with hypertension. PLoS Genet 6, e1001177, doi: 10.1371/journal.pgen.1001177 (2010).

43. Slavin, T. P., Feng, T., Schnell, A., Zhu, X. \& Elston, R. C. Two-marker association tests yield new disease associations for coronary artery disease and hypertension. Hum Genet 130, 725-733, doi: 10.1007/s00439-011-1009-6 (2011).

44. Wellcome Trust Case Control, C. Genome-wide association study of 14,000 cases of seven common diseases and 3,000 shared controls. Nature 447, 661-678, doi: 10.1038/nature05911 (2007).

45. Wang, Y. et al. EPHB4 Protein Expression in Vascular Smooth Muscle Cells Regulates Their Contractility, and EPHB4 Deletion Leads to Hypotension in Mice. J. Biol. Chem 290, 14235-14244, doi: 10.1074/jbc.M114.621615 (2015).

46. MacDonald, B. T., Tamai, K. \& He, X. Wnt/beta-catenin signaling: components, mechanisms, and diseases. Dev Cell 17, 9-26, doi: 10.1016/j.devcel.2009.06.016 (2009).

47. Gros, R. et al. GPR30 expression is required for the mineralocorticoid receptor-independent rapid vascular effects of aldosterone. Hypertension 57, 442-451, doi: 10.1161/HYPERTENSIONAHA.110.161653 (2011).

48. Bhattacharya, B. \& Roberts, R. E. Enhancement of alpha2-adrenoceptor-mediated vasoconstriction by the thromboxane-mimetic U46619 in the porcine isolated ear artery: role of the ERK-MAP kinase signal transduction cascade. Br. J. Pharmacol. 139, 156-162, doi: $10.1038 /$ sj.bjp.0705208 (2003).

49. Wang, Y. et al. Estrogen and testosterone in concert with EFNB3 regulate vascular smooth muscle cell contractility and blood pressure. Am J Physiol Heart Circ Physiol 310, H861-72, doi: 10.1152/ajpheart.00873 (2016).

50. Yujia, W. et al. EFNB2 deletion in smooth muscle cells results in hypotension in mice and EFNB2 mutation is associated with human hypertension risk. Eur J Human Genet (in press).

\section{Acknowledgements}

This work was supported by grants from the Canadian Institutes of Health Research to J.W. (MOP57697, MOP69089 and MOP 123389), H.L. (MOP97829), and G.C. (CMI72323). It was also financed by grants from the Natural Sciences and Engineering Research Council of Canada (203906-2012), and the J.-Louis Levesque Foundation to J.W. This study was also made possible by a group grant from the National Sciences Foundation of China (\#81361120264) to J.S., S.H. T.W. and J.W. The funders provided support in the form of salaries for authors [Y.W.; Z.W.; H.L.; J.P.; J.R.], and experimental costs, but did not have any additional role in the study design, data collection and analysis, decision to publish, or preparation of the manuscript. The specific roles of these authors are articulated in the "author contributions". The authors thank Regeneron Pharmaceuticals for generously providing Efnb3 KO mice. The authors thank all the authors of the International Consortium for Blood Pressure Genome-Wide Association Studies for allow us to mine the study dataset. 


\section{Author Contributions}

G.E., and P.M. were principal investigators of the ICBP genetic study from which the data used in this project are derived. Y.W., Z.W. J.P., and H.L. conducted mouse BP study in vivo and in vitro. J.R., and E.B and G.E. analyzed ICBP dataset. E.S., Z.Q. and G.C. carried out echocardiography. T.W., J.S. and S.H. studied human vascular gene expression. J.W. generated the concept and initiated this project. Y.W., J.R. and J.W. drafted the manuscript.

\section{Additional Information}

Supplementary information accompanies this paper at http://www.nature.com/srep

Competing financial interests: The authors declare no competing financial interests.

How to cite this article: Wang, Y. et al. The role of GRIP1 and ephrin B3 in blood pressure control and vascular smooth muscle cell contractility. Sci. Rep. 6, 38976; doi: 10.1038/srep38976 (2016).

Publisher's note: Springer Nature remains neutral with regard to jurisdictional claims in published maps and institutional affiliations.

(c) (i) This work is licensed under a Creative Commons Attribution 4.0 International License. The images or other third party material in this article are included in the article's Creative Commons license, unless indicated otherwise in the credit line; if the material is not included under the Creative Commons license, users will need to obtain permission from the license holder to reproduce the material. To view a copy of this license, visit http://creativecommons.org/licenses/by/4.0/

(C) The Author(s) 2016 